\title{
Sensitivity of echo enabled harmonic generation to sinusoidal electron beam energy structure
}

\author{
E. Hemsing, ${ }^{*}$ B. Garcia, Z. Huang, and T. Raubenheimer \\ SLAC National Accelerator Laboratory, Menlo Park, California 94025, USA
}

D. Xiang

Key Laboratory for Laser Plasmas (Ministry of Education), Department of Physics and Astronomy, Shanghai Jiao Tong University, Shanghai 200240, China

(Received 29 March 2017; published 19 June 2017)

\begin{abstract}
We analytically examine the bunching factor spectrum of a relativistic electron beam with sinusoidal energy structure that then undergoes an echo-enabled harmonic generation (EEHG) transformation to produce high harmonics. The performance is found to be described primarily by a simple scaling parameter. The dependence of the bunching amplitude on fluctuations of critical parameters is derived analytically, and compared with simulations. Where applicable, EEHG is also compared with high gain harmonic generation (HGHG) and we find that EEHG is generally less sensitive to several types of energy structure. In the presence of intermediate frequency modulations like those produced by the microbunching instability, EEHG has a substantially narrower intrinsic bunching pedestal.
\end{abstract}

DOI: $10.1103 /$ PhysRevAccelBeams.20.060702

\section{INTRODUCTION}

External seeding schemes like high gain harmonic generation (HGHG) [1] and echo-enabled harmonic generation (EEHG) $[2,3]$ have been developed to improve the spectral brightness and spectral stability of high-gain free electron lasers (FELs). These techniques use lasers and dispersive electron beam transport elements to generate high harmonic density modulations that jump-start the FEL amplification process and produce narrowband, fully coherent radiation down to $\mathrm{x}$-ray wavelengths.

The success of the seeding scheme, gauged in large part by the purity and stability of the final FEL output spectrum, depends sensitively on the details of the electron beam distribution. A primary contributor to deviations from ideal transform-limited FEL pulses are energy structures on the initial electron beam phase space. Uncontrolled energy structures lead to reductions in the spectral density (photons/eV) for FEL seeding systems because they are translated to the broader FEL output spectrum. Energy structures can occur as the result of collective effects during acceleration and compression [4] as in the case of coherent synchrotron radiation (CSR) [5-7] and the microbunching instability (MBI) [8-10], or transport effects such as wakefields. Wakefields typically have scale lengths comparable to the bunch length (tens of microns), whereas MBI

\footnotetext{
"ehemsing@slac.stanford.edu
}

Published by the American Physical Society under the terms of the Creative Commons Attribution 4.0 International license. Further distribution of this work must maintain attribution to the author(s) and the published article's title, journal citation, and DOI. can amplify broadband perturbations that end up peaked around $\gtrsim 1 \mu \mathrm{m}$ wavelengths. In either case, such energy structures can be difficult to remove completely, and they mix extra frequencies into the FEL gain bandwidth that can spoil the high-contrast, narrowband performance improvements offered by seeding in general. An illustrative example is the background pedestal observed in the selfseeding spectrum at LCLS [11-13]. At soft x-rays, the otherwise narrow seeded spike is surrounded by a background of frequencies that can contain a sizable portion (e.g., $\sim 40 \%$ ) of the power. For certain experiments, a final monochromator is needed to further filter the spectrum, but this comes with a limited efficiency $(\leq 10 \%)$ that significantly reduces the overall gains in spectral brightness.

Understanding these effects is important for evaluating HGHG and EEHG as competitive external seeding schemes, as they differ in their sensitivity to phase space structures. Linear energy-time chirps, for example, have the general effect of shifting the harmonic frequency away from the target frequency in HGHG, and EEHG is less sensitive to these effects [14]. Similarly, it has also been shown that the EEHG bunching bandwidth is less sensitive to quadratic chirps [15], which are analogous to linear laser chirps [16]. EEHG may also be more robust to energy structures like those that result from MBI [17], though the extent to which is not completely clear for lack of one-toone experimental comparisons, but simulation studies for upcoming FELs are ongoing $[18,19]$.

On the other hand, energy structures that can be precisely controlled offer additional tunability on the seeded FEL output. For example, small linear chirps can be used to shift the FEL frequency in a repeatable way [20]. Or, coherent modulations on the beam at IR wavelengths can produce 
discrete sidebands in the seeded FEL spectrum for dedicated multicolor operations [21].

Here we study EEHG analytically and with numerical simulations in an effort to shed light on some of these issues and establish the scaling and sensitivity. A general formalism is presented that introduces an energy modulation on the beam prior to the EEHG transformation. A simple scaling parameter is identified that governs the characteristics of the bunching spectrum for both EEHG and HGHG, and the bunching spectrum is then analyzed for two classes of idealized initial electron beam energy structure; long wavelength modulations that generate linear and quadratic chirps, and intermediate wavelength modulations shorter than the beam, such as those encountered in collective effects. EEHG is compared with simple HGHG to highlight the general distinctions and to provide a conceptual framework for more detailed future comparisons, particularly for ultrahigh harmonics that require cascading of multiple seeding stages.

\section{EEHG SCALING FACTOR AND OPTIMIZATION}

We closely follow the notation of [3] where the bunching factor for EEHG is given as,

$$
\bar{b}_{n, m}\left(k_{E}\right)=e^{-\xi_{E}^{2} / 2} J_{n}\left(-\xi_{E} A_{1}\right) J_{m}\left(-a_{E} A_{2} B_{2}\right),
$$

where the normalized laser modulations are $A_{1,2}=$ $\Delta E_{1,2} / \sigma_{E}$ and normalized dispersions are $B_{1,2}=$ $k_{1} R_{56}^{(1,2)} \sigma_{E} / E>0 . \sigma_{E}$ is the slice energy spread, and $E$ is the beam energy. We assume ideal lasers that completely cover the electron beam. The EEHG harmonic frequency is,

$$
k_{E}=a_{E} k_{1}=n k_{1}+m k_{2}
$$

with $a_{E}=n+m K$ the harmonic number, $n$ and $m$ integers, and $K=k_{2} / k_{1}$. The optimal $n$ is small and negative, and $m \gg 1$. We define the EEHG scaling factor as the parameter,

$$
\xi_{E}=a_{E} B-m K B_{1}=n B_{1}+a_{E} B_{2},
$$

where $B=B_{1}+B_{2}$. We will see that $\xi_{E}$ governs the performance of the EEHG bunching process, and it is nearly always advantageous to minimize $\left|\xi_{E}\right|$. For $\left|\xi_{E}\right|$ small, the ratio of the dispersions is approximately the harmonic number, $a_{E} \sim\left|n B_{1} / B_{2}\right|$ and typically $B_{1} \gg B_{2}$. The optimal value of $\xi_{E}$ is found from the maximum of $\left|e^{-\xi_{E}^{2} / 2} J_{n}\left(-\xi_{E} A_{1}\right)\right|$, and is given approximately by [22]:

$$
\xi_{E} \simeq \pm \frac{j_{n, 1}^{\prime}}{A_{1}}\left[1+\frac{1}{A_{1}^{2}} \frac{1}{1-\left(n / j_{n, 1}^{\prime}\right)^{2}}\right]^{-1}
$$

where $j_{n, 1}^{\prime} \approx|n|+0.81|n|^{1 / 3}$ is the first root of $J_{n}^{\prime}$. $\left|\xi_{E}\right|$ decreases like $1 / A_{1}$ for practical values, so a larger $A_{1}$ is generally preferable for the bunching performance, with the constraint that it (and $A_{2}$ combined) not be so large as to strongly inhibit FEL amplification. In EEHG the scaling factor $\xi_{E}$ can also be negative. This carries several advantages [17,23], and it affects, for example, the tunable region of excited sideband frequencies (Sec. IV B).

$A_{1}$ determines the optimal $\xi_{E}$, so for a given $A_{2}$, the optimal dispersion values are then,

$$
B_{2}=\frac{j_{m, 1}^{\prime}}{a_{E} A_{2}}, \quad B_{1}=\left(\xi_{E}-a_{E} B_{2}\right) / n .
$$

Figure 1 shows the dependence of $\left|\bar{b}_{n, m}\left(k_{E}\right)\right|$ on the dispersions. The bunching factor peaks at two values of $B_{1}$, the larger of which is in the preferred $\xi_{E}<0$ region.

HGHG, in contrast, uses only one modulator and chicane to produce the harmonic frequency $k_{H}=a_{H} k_{1}$. The bunching factor is given by, $\bar{b}_{a_{H}}\left(k_{H}\right)=e^{-\xi_{H}^{2} / 2} J_{a_{H}}\left(-\xi_{H} A_{1}\right)$, where the HGHG scaling factor is,

$$
\xi_{H}=a_{H} B_{1}>0
$$

Analogous to $\xi_{E}, \xi_{H}$ governs key performance aspects of the HGHG process. Equation (4) also gives the optimum for $\xi_{H}$ with the replacement $n=a_{H} \gg 1$. The maximal harmonic bunching with the smallest energy spread yields $A_{1} \approx a_{H}$, for which the optimization is $\xi_{H} \approx 1$. Compare this with EEHG where, for example, with $A_{1}=3$ and $n=-1$, the optimum gives $\left|\xi_{E}\right| \approx 1 / 2$. This difference between the scaling parameters $\xi_{E}$ and $\xi_{H}$ accounts for several distinctions between the two schemes.

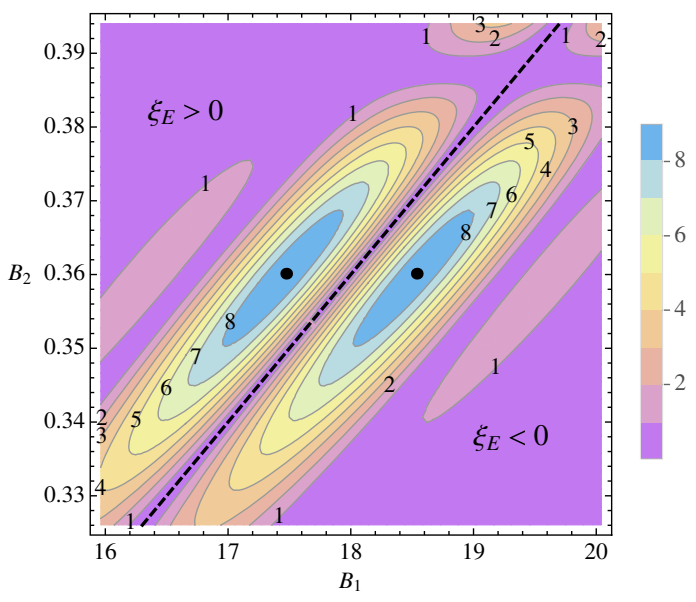

FIG. 1. $\left|\bar{b}_{n, m}\left(k_{E}\right)\right| \%$ for $a_{E}=50$ th harmonic of $260 \mathrm{~nm}$ lasers with $n=-1$ and $A_{1,2}=3$. The dashed line is $\xi_{E}=0$, and the $\xi_{E}= \pm 1 / 2$ optima (dots) lie at the centers of the bunching peaks in their respective regions, with a difference in dispersion $\Delta B_{1}=-2 \xi_{E} / n$. 


\section{A. Bunching sensitivity}

The EEHG bunching factor can be expanded about small deviations from the optimal tune parameters $B_{1,2}$ and $A_{1,2}$ to reveal how the bunching amplitude is affected. The impact of small changes in the first dispersion $\Delta B_{1}$ can be calculated by expansion about the value in (5). To second order, the bunching decreases as

$$
\begin{aligned}
\frac{\Delta \bar{b}_{n, m}}{\bar{b}_{n, m}} & =-\frac{\left(\Delta B_{1}\right)^{2}}{2 B_{1}^{2}}\left(\frac{n B_{1}}{\xi_{E}}\right)^{2}\left[\xi_{E}^{2}\left(A_{1}^{2}+\xi_{E}^{2}+2\right)-n^{2}\right] \\
& \simeq-\frac{\left(\Delta B_{1}\right)^{2}}{2 B_{1}^{2}}\left(j_{m, 1}^{\prime} \frac{A_{1}}{A_{2}}\right)^{2}\left[1-n^{2} /\left(j_{n, 1}^{\prime}\right)^{2}\right] .
\end{aligned}
$$

The second expression is the expansion for small $\xi_{E}$. From this we see that the bunching change is only weakly sensitive to $n$, but scales like $\left(\Delta B_{1} / B_{1}\right)^{2} m^{2}$, and thus is increasingly more sensitive to high harmonics $m \gg 1$. The effect is shown in Fig. 2. Small changes in the second dispersion $\Delta B_{2}$ yield a similar form, but with an additional term,

$$
\begin{aligned}
\frac{\Delta \bar{b}_{n, m}}{\bar{b}_{n, m}} \simeq & -\frac{\left(\Delta B_{2}\right)^{2}}{2 B_{2}^{2}}\left[\left(j_{m, 1}^{\prime} \frac{A_{1}}{A_{2}}\right)^{2}\left[1-n^{2} /\left(j_{n, 1}^{\prime}\right)^{2}\right]\right. \\
& \left.+\left[\left(j_{m, 1}^{\prime}\right)^{2}-m^{2}\right]\right] .
\end{aligned}
$$

For large harmonics, the second term scales like $m^{4 / 3}$ and can generally be neglected, so relative changes $\Delta B_{1} / B_{1}$ and $\Delta B_{2} / B_{2}$ reduce the bunching according to essentially the same scaling.

Similarly, if the first laser modulation fluctuates about the value $A_{1}$, the bunching amplitude varies as:

$$
\frac{\Delta \bar{b}_{n, m}}{\bar{b}_{n, m}}=\xi_{E}^{2} \frac{\Delta A_{1}}{A_{1}}-\frac{\left(\Delta A_{1}\right)^{2}}{2 A_{1}^{2}}\left[\xi_{E}^{2}\left(1+A_{1}^{2}\right)-n^{2}\right] .
$$

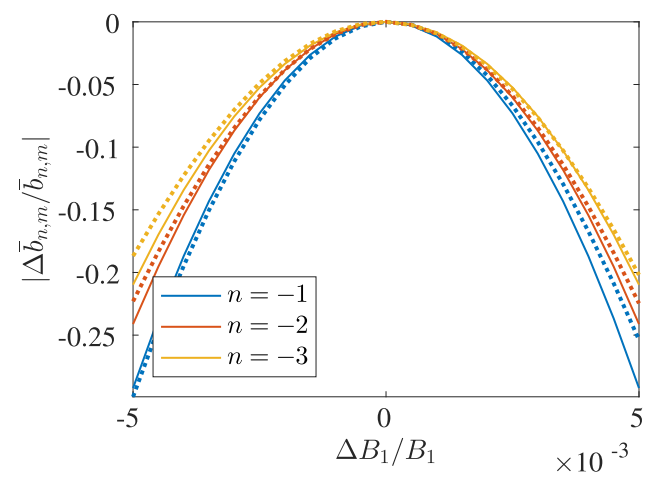

FIG. 2. Variation of bunching with deviations in $B_{1}$ from the approximate optimum in Eq. (4). $A_{1}=5, A_{2}=3, a_{E}=100$ is assumed. Solid lines are from Eq. (7), dashed lines are from exact solutions.

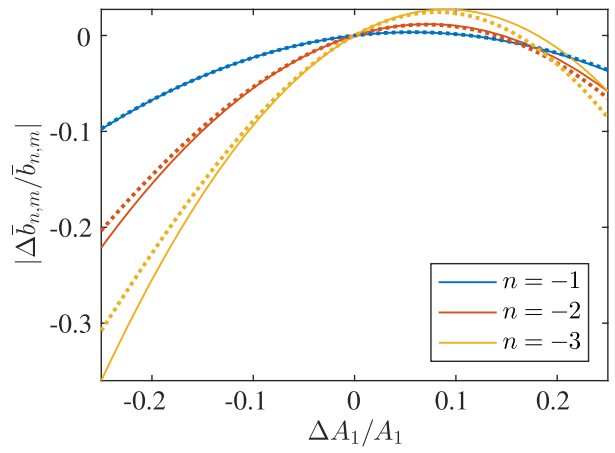

FIG. 3. Variation of bunching with deviations in $A_{1}$ from that used to calculate Eq. (4). $A_{1}=5$ is assumed. Solid lines are from Eq. (9), dashed lines are from exact solutions.

This is plotted in Fig. 3. There is a linear dependence on $\Delta A_{1} / A_{1}$ because the optimal energy modulation for a fixed $\xi_{E}$ is $A_{1} \simeq j_{n, 1}^{\prime} / \xi_{E}$, which is slightly larger than the nominal value used to calculate the optimal $\xi_{E}$. Clearly, the bunching in tunes with larger $|n|$ is more sensitive to $\Delta A_{1} / A_{1}$. This can impact the performance of an EEHG seeding system that, for example, uses a larger $|n|$ value in exchange for a smaller $B_{1}$.

Both Eqs. (7) and (9) deal with variations in the first modulator and chicane, so they also then apply directly to HGHG for the corresponding parameters with $\xi_{E} \rightarrow \xi_{H}$, and with $n \rightarrow a_{H}$.

A similar analysis applies for variation in the second EEHG modulation from the optimum in Eq. (5),

$$
\begin{aligned}
\frac{\Delta \bar{b}_{n, m}}{\bar{b}_{n, m}} & =-\frac{\left(\Delta A_{2}\right)^{2}}{2 A_{2}^{2}}\left[\left(j_{m, 1}^{\prime}\right)^{2}-m^{2}\right], \\
& \simeq-0.81 \frac{\left(\Delta A_{2}\right)^{2}}{A_{2}^{2}} m^{4 / 3} .
\end{aligned}
$$

Figure 4 shows the effect that amplitude variations in the second modulator have on the bunching. Higher harmonics are increasingly more sensitive, and the bunching is

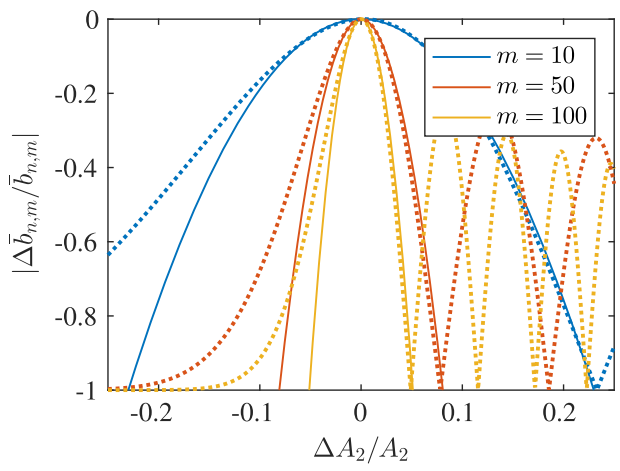

FIG. 4. Variation of bunching with deviations in $A_{2}$ from $j_{m, 1}^{\prime} / a B_{2}$. Solid lines are from Eq. (7), dashed lines are from exact solutions. 
strongly suppressed when $\Delta A_{2} / A_{2} \simeq 1 / \mathrm{m}^{2 / 3}$. For example, it only takes a 5\% increase in $A_{2}$ to kill the bunching at $m=100$. A comparison with Fig. 3 confirms that the EEHG bunching is much more sensitive to amplitude variations in the second modulator than in the first modulator.

\section{LONG WAVELENGTH MODULATIONS}

Let us consider the effect of initial energy structures on the beam that have long wavelengths compared to the bunch length, $\lambda_{0}=2 \pi / k_{0} \gg 2 \pi \sigma_{z}$. We define $h_{1}$ and $h_{2}$ as the dimensionless linear and quadratic coefficients of the energy variation,

$$
p_{0}=p+h_{1} k_{1} z+h_{2} k_{1}^{2} z^{2}
$$

where $p=\Delta E / \sigma_{E}$ is the normalized energy variable, $z$ is the coordinate in the beam, and

$$
h_{1}=\left.\frac{1}{k_{1}} \frac{d p_{0}}{d z}\right|_{z=0}, \quad h_{2}=\left.\frac{1}{2 k_{1}^{2}} \frac{d^{2} p_{0}}{d z^{2}}\right|_{z=0} .
$$

Previous analyses have computed the bunching factor spectrum for purely linear chirps [14], and independently for purely quadratic curvature [16].

The bunching spectrum for frequencies near $k_{E}$ is then (see Appendix),

$$
b_{n, m}(k)=\bar{b}_{n, m}(k) \frac{\exp \left[-\frac{\sigma_{z}^{2}\left(k-n k_{1}-m k_{2}+\xi h_{1} k_{1}\right)^{2}}{2(1+i \chi)}\right]}{\sqrt{1+i \chi}},
$$

where

$$
\xi=\frac{k}{k_{1}} B-m K B_{1},
$$

is the generalized scaling parameter at the frequency $k$, (where $\xi\left(k_{E}\right)=\xi_{E}$ ) and

$$
\bar{b}_{n, m}(k)=e^{-\xi^{2} / 2} J_{n}\left(-\xi A_{1}\right) J_{m}\left(-k A_{2} B_{2} / k_{1}\right) .
$$

This is the bunching spectrum envelope. It is the spectral response function of the EEHG transformation as dictated by the tune, which is set by the scaled energy modulations and dispersive strengths.

We have also defined the quadratic chirp parameter

$$
\chi(k)=2 \xi(k) h_{2} k_{1}^{2} \sigma_{z}^{2}
$$

The relative energy spread of the beam in Eq. (11) is $\sigma_{p_{0}}^{2}=1+\left(h_{1} k_{1} \sigma_{z}\right)^{2}+3\left(h_{2} k_{1}^{2} \sigma_{z}^{2}\right)^{2}$, so $\chi$ may be interpreted as the magnitude of the quadratic energy chirp over the whole beam, scaled by $\xi(k)$.

\section{A. Harmonic shift}

The bunching in (13) has a sharp peak at $k=n k_{1}+m k_{2}-\xi h_{1} k_{1}$. With Eq. (14), the new EEHG harmonic $a$ is located at [14],

$$
a=\frac{n+m K\left(1+h_{1} B_{1}\right)}{1+h_{1} B} .
$$

The linear chirp $h_{1}$ shifts the harmonic $a$ away from the unchirped harmonic $a_{E}$ in Eq. (2). The chirp also shifts the scaling parameter to $\xi\left(a k_{1}\right)=\xi_{E} /\left(1+h_{1} B\right)$, which can be used with Eq. (7) and with $\Delta \xi_{E}=n \Delta B_{1}$ to estimate the impact of $h_{1}$ on the bunching. In general, $\left|h_{1}\right| \ll 1$, so expanding to first order gives $a \approx a_{E}-h_{1} \xi_{E}$ The same analysis can be applied for HGHG to yield $a \approx a_{H}-h_{1} \xi_{H}$, and measurement of tunable shifts in output wavelength have been reported in [24]. Thus the relative shift of the central frequency in the presence of a linear chirp between the two schemes is

$$
\frac{(\Delta a)_{E E H G}}{(\Delta a)_{H G H G}} \approx \frac{\xi_{E}}{\xi_{H}} .
$$

The relative shift depends only on the scaling factors for each scheme, and EEHG is generally less sensitive because $\left|\xi_{E}\right|<\xi_{H}$. Consider the aforementioned example where $A_{1}=3$ and $n=-1$, which gives $\left|\xi_{E}\right| \approx 1 / 2$, compared with $\xi_{H} \approx 1$ for HGHG. Note however that for $n=-2$, then $\left|\xi_{E}\right| \approx 0.85$ and they become comparable. We also see that for a fixed chirp $h_{1}$, the direction of the central frequency shift in EEHG depends on the sign of $\xi_{E}$ [23].

\section{B. Cascaded HGHG}

This model can be used to examine the harmonic shift from a linear chirp in EEHG compared with two-stage HGHG for high harmonic bunching. The bunching harmonic from the first stage of a cascaded HGHG setup is $a_{H}^{(1)}-h_{1}^{(1)} \xi_{H}^{(1)}$. The first stage harmonic, linear chirp, and scaling factor are labeled by the superscript. The bunched beam then radiates coherent light at this shifted harmonic frequency that, after some delay, modulates the beam again. Because cascaded HGHG utilizes fresh bunch seeding, we allow for the possibility that the chirp is different between the different parts of the beam and for the moment, that the scaling factor can also vary. After dispersion this second modulation produces bunching at a higher harmonic,

$$
a \approx a_{H}^{(2)}\left(a_{H}^{(1)}-h_{1}^{(1)} \xi_{H}^{(1)}\right)-h_{1}^{(2)} \xi_{H}^{(2)} .
$$

For the sake of comparison with EEHG, the goal is to reach the same final harmonic by both methods, so $a_{E}=a_{H}^{(2)} a_{H}^{(1)}$. An interesting feature of cascaded HGHG is that the shift in the harmonic away from $a_{H}^{(2)} a_{H}^{(1)}$ could be zeroed out if the contributions from the chirp in the second 
bunch cancel those from the first, namely, when $h_{1}^{(2)} \xi_{H}^{(2)}=-a_{H}^{(2)} h_{1}^{(1)} \xi_{H}^{(1)}$.

Assuming that the scaling factors in each HGHG stage are $\operatorname{similar}\left(\xi_{H}^{(1)} \approx \xi_{H}^{(2)}\right)$, the harmonic shift of HGHG compared with EEHG on the same linearly chirped beam is

$$
\frac{(\Delta a)_{E E H G}}{(\Delta a)_{C-H G H G}} \approx \frac{\xi_{E}}{a_{H}^{(2)} \xi_{H}}
$$

We see that, as opposed to the single stage HGHG case in Eq. (18), even if the scaling factors $\xi_{E}$ and $\xi_{H}$ are similar in magnitude, the harmonic shift of the two-stage HGHG process is more pronounced due to the amplification of the shift from the first stage by the harmonic jump in the second stage. From this point of view, the final central wavelength shift of cascaded HGHG is reduced by performing a larger harmonic jump in the first stage to reduce $a_{H}^{(2)}$.

\section{Harmonic bandwidth}

The bandwidth of the narrow harmonic spike at $a k_{1}$ is determined by $\left|b_{n, m}(k)\right|^{2}$ in Eq. (13). Assuming $\left|\bar{b}_{n, m}(k)\right|^{2}$ is essentially constant, the relative rms bandwidth $\sigma=\sigma_{k} / a k_{1}$ of the bunching spike is approximately

$$
\sigma=\sigma_{0} \frac{\left[1+\left(\frac{\chi_{E}}{1+h_{1} B}\right)^{2}\right]^{1 / 2}}{1+m K h_{1} B_{1} / a_{E}}
$$

where $\chi_{E}=\chi\left(k_{E}\right)=2 \xi_{E} h_{2} k_{1}^{2} \sigma_{z}^{2}$, and $\sigma_{0}=1 /\left(\sqrt{2} \sigma_{z} k_{E}\right)$ is the relative rms bandwidth of the harmonic spike in the absence of any initial energy structure on the beam.

We consider the behavior of the bunching bandwidth due to the linear and quadratic chirps separately. When the quadratic component vanishes $\left(h_{2}=0\right)$ and the beam has only a linear chirp, the calculated relative bandwidth with EEHG is then,

$$
(\sigma)_{E E H G}=\frac{\sigma_{0}}{1+m K h_{1} B_{1} / a_{E}} \quad\left(h_{2}=0\right) .
$$

We see that it depends on product of the dispersion in the first chicane $B_{1}$ and the harmonic of the second laser $m$. HGHG only uses one laser and one chicane, so this term vanishes for HGHG:

$$
(\sigma)_{H G H G}=\sigma_{0}, \quad\left(h_{2}=0\right) .
$$

In HGHG, the change in bandwidth due to linear beam compression (or decompression) is offset by the identical shift in frequency, so the relative bandwidth is unaffected by the linear chirp. In contrast, the EEHG frequency shift doesn't correspond to the bunch length change through the large dispersion. Accordingly, with a positive linear chirp $h_{1}>0$ the bandwidth is smaller than when the beam has no chirp at all. The trends are shown in Figure 5, which includes cases of atypically large chirps to highlight the effect (e.g, $\left|h_{1}\right|=0.16$ corresponds to an energy chirp of $\sigma_{E}$ per laser wavelength $\lambda_{1}$ ). The denominator of Eq. (22) is essentially the bunch compression factor in the first EEHG chicane with $m K h_{1} B_{1} / a_{E} \simeq h_{1} B_{1}$, so this effect is negligible when the change in bunch length is small, $\left(\left|h_{1} B_{1}\right| \ll 1\right)$.

When the linear component of the chirp vanishes $\left(h_{1}=0\right)$ and the beam has only a quadratic chirp, the scaled bandwidth is described by two regimes. The small curvature regime $\left|h_{2} k_{1}^{2} \sigma_{z}^{2}\right|<1$ is satisfied when the curvature over the beam is on the order of the slice energy spread or less. The bandwidth then scales like $\sigma / \sigma_{0} \approx 1+\chi_{E}^{2} / 2$. The comparative increase in relative bandwidth $\Delta \sigma=\sigma-\sigma_{0}$ due to $h_{2}$ in this regime is then,

$$
\frac{(\Delta \sigma)_{E E H G}}{(\Delta \sigma)_{H G H G}}=\left(\frac{\xi_{E}}{\xi_{H}}\right)^{2}, \quad\left(h_{1}=0,\left|\chi_{E}\right|<1\right) .
$$

Perhaps more practically relevant is the $\left|\chi_{E}\right|>1$ regime, which can occur if the quadratic component of energy variation over the beam is larger than the slice energy spread, $\left|h_{2} k_{1}^{2} \sigma_{z}^{2}\right|>1$. In this case, the relative bandwidth is $\sigma / \sigma_{0} \approx\left|\chi_{E}\right|$ and,

$$
\frac{(\sigma)_{E E H G}}{(\sigma)_{H G H G}}=\left|\frac{\xi_{E}}{\xi_{H}}\right|, \quad\left(h_{1}=0,\left|\chi_{E}\right|>1\right) .
$$

Note from Eq. (13) that $\left|b_{n, m}\right|^{2}$ is reduced in this regime by the factor $\left|\chi_{E}\right|$.

Figure 6 shows the relative bandwidth growth of HGHG and EEHG due to $h_{2}$, where again HGHG is approximately

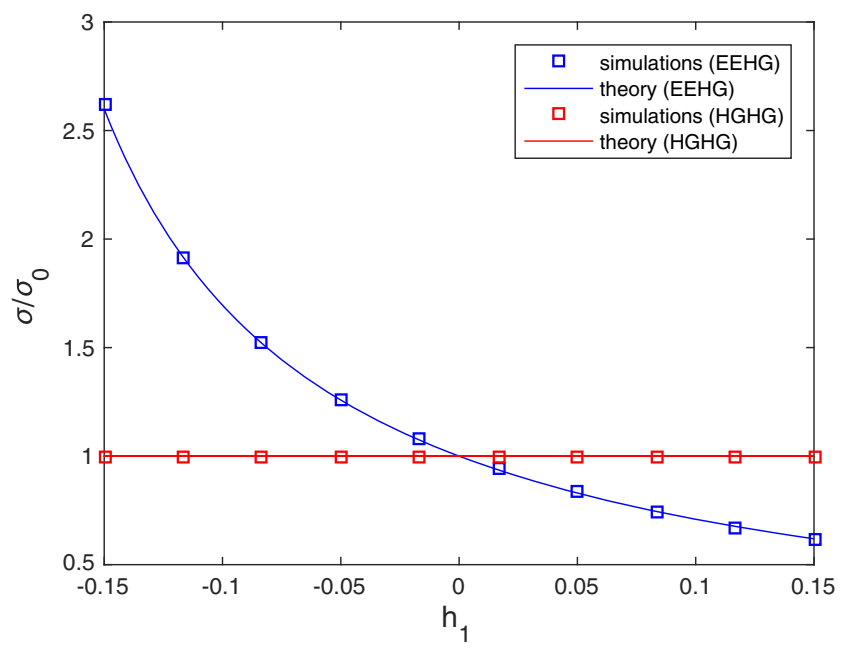

FIG. 5. Harmonic bunching bandwidth as a function of linear chirp. For EEHG $a_{E}=10, m=11, K=1, B_{1}=3.73$, and $A_{1,2}=3$. For HGHG $a_{H}=A_{1}=10$ and $B_{1}=0.11$. Numerical particle simulations written in MATLAB [25]. 


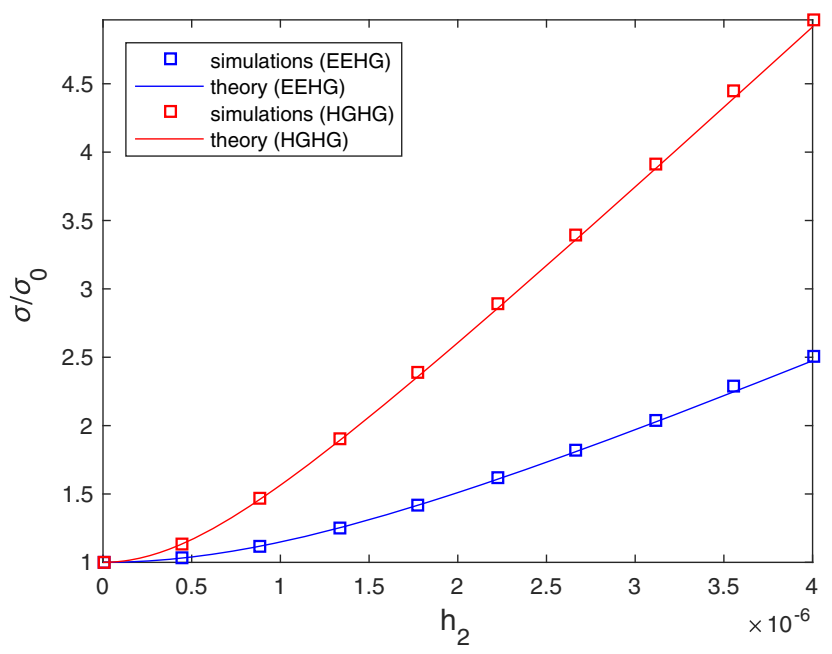

FIG. 6. Harmonic bunching bandwidth as a function of quadratic chirp. Same parameters as Fig. 5.

twice as sensitive to the quadratic curvature because $\xi_{H} \approx 2 \xi_{E}$. The relative insensitivity of EEHG to quadratic curvature has also been verified experimentally [15].

Finally, it is interesting to point out that if $\xi_{E}$ is sufficiently small, it is possible to be in the large curvature regime $\left|h_{2} k_{1}^{2} \sigma_{z}^{2}\right|>1$ but for $\left|\chi_{E}\right|<1$ so that the bandwidth scales quadratically with $\xi_{E}$. This is another case where small values of $\xi_{E}$ (and $\xi_{H}$ ) can aid in mitigating undesirable effects.

\section{INTERMEDIATE WAVELENGTH MODULATIONS}

Intermediate wavelength modulations are short compared to the bunch but long compared to the laser wavelengths, $\lambda_{1}, \lambda_{2} \ll \lambda_{0} \ll 2 \pi \sigma_{z}$. These are of practical concern specifically for MBI and/or high frequency wakefield effects. In particular, MBI in modern beams can produce strong initial energy modulations in the beam at micron wavelength scales which can then produce sidebands in the bunching spectrum. Because the MBI is a broadband instability, a large range of excited sidebands can be amplified by the FEL if they are within the FEL gain bandwidth. EEHG and HGHG have different sensitivity to such structures due to both their respective scaling factors, and their excitation bandwidths.

\section{A. Excitation bandwidth}

The excitation bandwidth (EB) is defined here as the width of the bunching spectrum envelope $\bar{b}_{n, m}(k)$ in (15). Shown in Fig. 7 for EEHG, it specifies a frequency region around the target harmonic wherein sidebands can be generated in the bunching spectrum during the harmonic up conversion process. Inspection of Eq. (15) shows that the EB is determined by the Gaussian suppression factor $e^{-\xi^{2} / 2}$. We can therefore approximate the extent of the EB

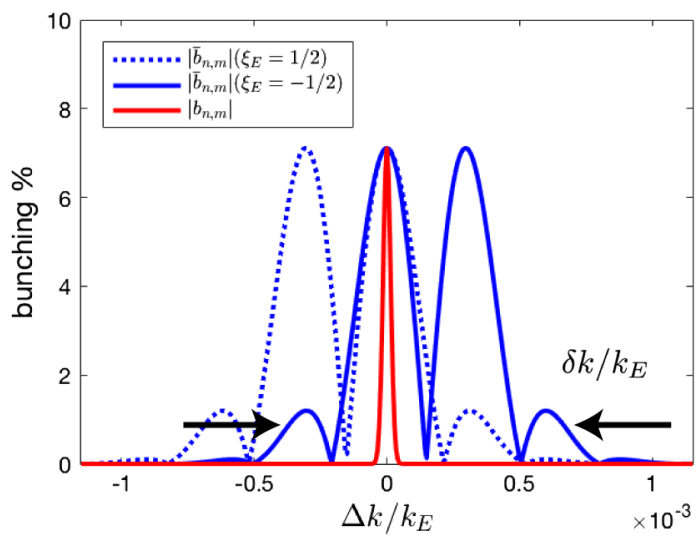

FIG. 7. Harmonic bunching spectrum envelopes (blue) of EEHG, and bunching harmonic spike (red), for $a_{E}=100$ with $A_{1,2}=3, n=-1$. The excitation bandwidth $\delta k / k_{E}$ is the width of the envelope, which shifts position depending on the sign of optimal $\xi_{E}$.

by considering the values of $\xi$ (and therefore $k$ ) that may still lead to bunching. For EEHG we define a full bandwidth bounded by $-2 \leq \xi \leq 2$, which gives

$$
\left(\frac{\delta k}{k_{E}}\right)_{E E H G}=\frac{4}{a_{E} B} \approx\left|\frac{4 n A_{2}}{a_{E}^{2}}\right|
$$

We have assumed $B \approx\left|a_{E} B_{2} / n\right|$ and $B_{2} \approx 1 / A_{2}$ in the last step. Initial modulation frequencies $k_{0}=2 \pi / \lambda_{0}<\delta k / 2$ sit within the excitation bandwidth and may be excited as bunching sidebands at $k_{E} \pm k_{0}$ during the EEHG process. These sidebands are coherently amplified and grow at the same rate as the harmonic during FEL lasing if they are within the FEL bandwidth. In contrast, frequencies $k_{0}>$ $\delta k / 2$ are outside the cutoff and are suppressed. We can thus relate the EB directly to the bandwidth of the coherently excited spectral pedestal in EEHG. From the scaling in (26) it is desirable to operate with the largest value of $B$ (or smallest $A_{2}$ ) to narrow the pedestal, which corresponds to the smallest $|n|$ tune for a given harmonic.

A potentially powerful feature of the EEHG EB is that it can be made smaller than the saturated bandwidth $\sim \rho$ [26] of a high-gain FEL in order to narrow the pedestal of excited frequencies. Consider a harmonic of $a_{E}=100$, with $A_{2}=3$ and $n=-1$, for which $\delta k / k_{E} \approx 0.12 \%$. This is smaller than the full bandwidth of most modern soft x-ray FELs.

We are compelled to define a similar excitation bandwidth for HGHG, but it turns out that the much weaker dispersion does not offer much damping of the sidebands. Using the previous logic, the HGHG EB can be defined by the bounds $0<\xi=\frac{k}{k_{1}} B_{1} \leq 2$, which gives $\left(\delta k / k_{H}\right)_{H G H G} \sim 2 / a_{H} B_{1}=2 / \xi_{H}$. But because $\xi_{H}<2$ for sufficient bunching, this describes a broadband EB of order unity, 


$$
\left(\frac{\delta k}{k_{H}}\right)_{H G H G} \sim \mathcal{O}(1)
$$

Whether the sidebands are in fact excited depends on their modulation amplitude and frequency, but in contrast to EEHG, there is no natural pedestal suppression or narrowing furnished by the HGHG process.

\section{B. Sideband bunching amplitudes}

With the definition of the bunching envelope and excitation bandwidth (see Fig. 7), we can now consider how sidebands and spectral pedestals may be excited in EEHG. Consider a monochromatic energy modulation with amplitude $A_{0}$ and frequency $k_{0}$ on the e-beam prior to the EEHG transformation,

$$
p_{0}=p+A_{0} \sin \left(k_{0} z\right)
$$

The bunching at the different discrete sideband frequencies $k_{E}+q k_{0}$, can be calculated from EEHG theory [see Eq. (A5) in the Appendix]. We presume the simple case where there is no spectral overlap between the bunching peaks for the different sidebands and their harmonics $q$, so the bunching factor at each frequency is given by the corresponding Bessel function $J_{q}$.

The bunching at the target EEHG harmonic $k_{E}$ is then simply given by,

$$
b_{n, m}\left(k_{E}\right)=\bar{b}_{n, m}\left(k_{E}\right) J_{0}\left(-\xi_{E} A_{0}\right) .
$$

This is just the standard EEHG bunching factor modified by the $J_{0}$ term of the initial energy modulation for $q=0$. Shown in Fig. 8, the bunching at $k_{E}$ is reduced for $A_{0}>0$, and it is completely suppressed whenever $A_{0}=j_{0, l} / \xi_{E}$, where $j_{0, l}$ is the $l^{\text {th }}$ root of $J_{0}$. Equation (29) also applies for HGHG, and because $\left|\xi_{E}\right|<\xi_{H}$, the EEHG bunching amplitude is more robust to small initial energy modulations. The reduction of the harmonic bunching is a broadband effect because Eq. (29) is independent of $k_{0}$. Note that the bunching at $k_{E}$ varies strongly as a function of $A_{0}$ according to the behavior of $J_{0}$. As such, for large $A_{0}$, it is possible to still produce harmonic bunching in a beam where the laser modulations are smaller than the initial modulation.

The bunching at the nearest sideband frequencies $k_{ \pm}=k_{E} \pm k_{0}$ is

$$
b_{n, m}\left(k_{ \pm}\right)=\bar{b}_{n, m}\left(k_{ \pm}\right) J_{ \pm 1}\left(-\xi\left(k_{ \pm}\right) A_{0}\right),
$$

where $\xi\left(k_{ \pm}\right)=\xi_{E} \pm k_{0} B / k_{1}$. The EEHG sideband bunching spectrum in (30) is highly sensitive to $k_{0}$ and $A_{0}$, in part because the bunching envelope has strong amplitude variations within the excitation bandwidth. For example, modulations at frequencies near zeros of the bunching envelope $k_{0}=-\left(k_{1} / B\right)\left( \pm j_{n, l} / A_{1}+\xi_{E}\right)$ produce weak
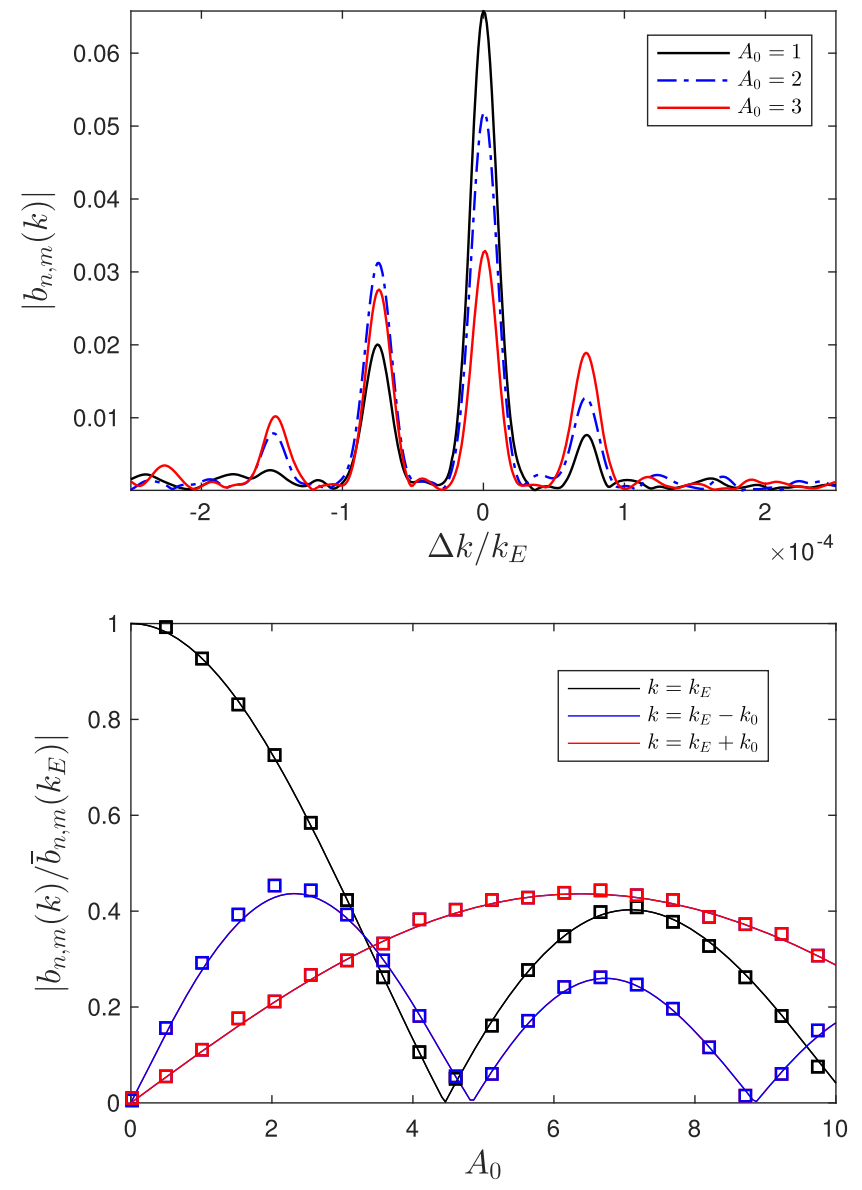

FIG. 8. Top: Bunching and sideband spectra for different $A_{0}$ with $a_{E}=100, A_{1,2}=3, n=-1$, and $k_{0}=\left|k_{1} \xi_{E} / 2 B\right|$. Bottom: Bunching amplitudes as a function of $A_{0}$. Solid lines are predictions from the analytic model, squares are from numerical simulations.

bunching sidebands, while those at in-between frequencies where it is peaked can produce strong bunching.

Figure 8 also shows how specific $k_{E} \pm k_{0}$ sideband amplitudes near $k_{E}$ vary with $A_{0}$. The dependence is not symmetric about $k_{E}$; here the high frequency sideband $(q=1)$ is smaller than the low frequency sideband $(q=-1)$ for $A_{0}<3$. The opposite would be true for the $\xi_{E}>0$ tune.

A tunable $A_{0}$ may provide a way to simultaneously select or suppress different frequencies. As seen in the lower plot of Fig. 8 for example, at $A_{0} \approx 3$ the bunching at $k_{E}$, $k_{E}+k_{0}$, and $k_{E}-k_{0}$ are all comparable, whereas for $A_{0} \approx 4.5$ the bunching at $k_{E}+k_{0}$ dominates. This feature may have direct applications in tunable multicolor FELs for shifting the power between different colors.

If $k_{0} \ll\left|k_{1} \xi_{E} / B\right|$, the sidebands are very close to the harmonic $k_{E}$ and can become comparable in amplitude. The ratio is,

$$
\frac{b_{n, m}\left(k_{ \pm}\right)}{b_{n, m}\left(k_{E}\right)} \approx \mp \frac{J_{1}\left(A_{0} \xi_{E}\right)}{J_{0}\left(A_{0} \xi_{E}\right)}
$$


They are approximately equal when $\left|A_{0} \xi_{E}\right| \simeq 1.4$, so the energy modulation must satisfy

$$
A_{0}<\left|1.4 / \xi_{E}\right|
$$

to keep the sideband bunching smaller than the harmonic bunching. If so, we obtain the approximate relation,

$$
\frac{b_{n, m}\left(k_{ \pm}\right)}{b_{n, m}\left(k_{E}\right)} \approx \mp \frac{A_{0} \xi_{E}}{2}\left(1+\frac{\left(A_{0} \xi_{E}\right)^{2}}{8}\right) .
$$

From the explicit dependence on the scaling parameter in this expression, we conclude that the contrast between the harmonic bunching at $k_{E}$ and the nearest sidebands at $k_{E} \pm k_{0}$ is about twice as high in EEHG than in HGHG for the same $A_{0}$. The EEHG bunching spectrum therefore appears to be more immune to sideband excitations.

\section{Broadband modulations}

The single frequency initial energy modulations in this analytic model agree well with numerical simulations of the bunching. Realistic beams, however, often have energy structures that span a broad range of wavelengths.

Figure 9 shows the simulation results of EEHG on a beam that has a broad spectrum of energy modulations similar to those encountered from the MBI. The width of the plotted window is defined the EB given by Eq. (26). The spectrum of MBI energy structure is modeled to mimic the general microbunching gain spectrum due to longitudinal space charge (e.g., [27]), and has the form $p_{0}=p+C \sum_{i}^{N} \delta_{i}^{2} \exp \left(-\delta_{i}^{2}\right) \sin \left(\delta_{i} k_{0} z+\phi_{i}\right)$, where $N$ is the number of individual modulations (here 40 ), $\delta_{i}$ is the frequency factor, and $\phi_{i}$ is a random phase. The initial modulation spectrum is peaked at the wavelength $\lambda_{0}=$ $3 \mu \mathrm{m}$ here, and the value of the amplitude $C$ is chosen to adjust the integrated energy spread $\sigma_{p_{0}}$ after the modulations for the different cases. A range of frequencies for $\delta_{i}$ is chosen that extends well into the high $\left(\ll \lambda_{0}\right)$ and low $\left(\gg \lambda_{0}\right)$ cutoff regions, with $\delta_{i}$ picked randomly from within each of the $N$ evenly divided spectral portions. As $N$ becomes large, the energy kick on each electron is effectively statistical, and the bunching at the harmonic is predicted to be attenuated by the factor $e^{-\xi_{E}^{2}\left(\sigma_{P_{0}}^{2}-1\right) / 2}[22]$. This is confirmed quantitatively by the steady reduction of the peak.

As $\sigma_{p_{0}}$ increases from the noisy modulations, a growing pedestal is observed surrounding the central harmonic. The pedestal spectrum fluctuates in shape in each simulation owing to its simulated MBI origin, but the general EEHG envelope is clearly revealed in the emergence of specific regions of pedestal growth over multiple simulation runs, particularly for larger $\sigma_{p_{0}}$. The fraction of power (in $\left.\left|b_{n, m}(k)\right|^{2}\right)$ as a function of bandwidth is also plotted. From this example and in general, the contrast between the
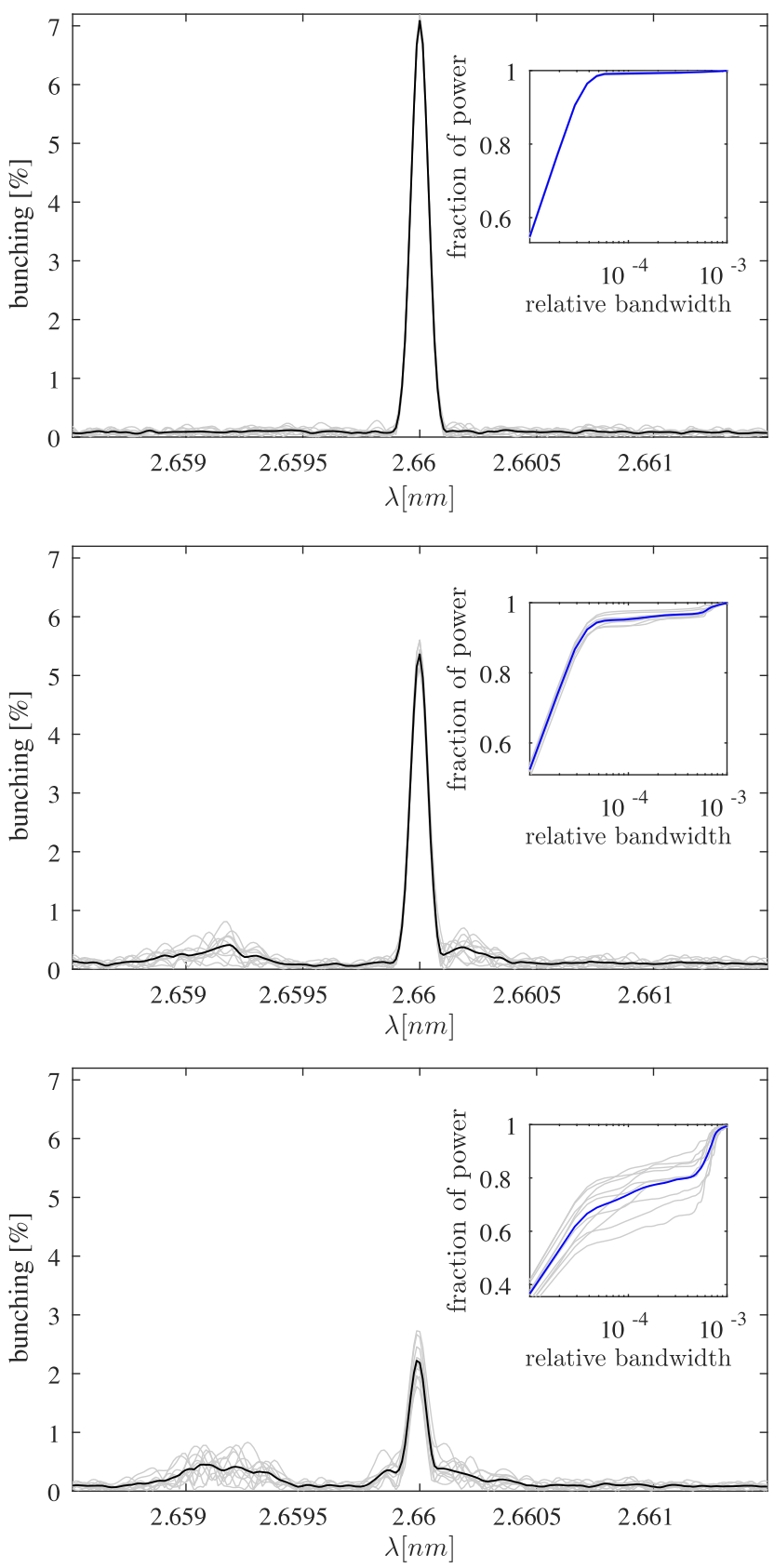

FIG. 9. EEHG bunching simulations for the $a_{E}=100$ harmonic of $266 \mathrm{~nm}$ lasers in the presence of broadband MBI energy modulations with rms energy spread (a) $\sigma_{p_{0}}=1$, (b) $\sigma_{p_{0}}=\sqrt{3}$, (c) $\sigma_{p_{0}}=3$. The beam is modeled after the parameters of LCLSII; $E=4 \mathrm{GeV}, \sigma_{E}=500 \mathrm{keV}$, and $\sigma_{z} / c=100 \mathrm{fs}\left(\sigma_{0}=10^{-5}\right)$. The EEHG energy modulations are $A_{1,2}=3$ and the dispersions are $R_{56}^{(1)}=12 \mathrm{~mm}\left(B_{1}=35.46\right)$ and $R_{56}^{(2)}=118 \mu \mathrm{m}$ $\left(B_{2}=0.35\right)$. At these settings $\xi_{E}=-0.53$.

harmonic peak and pedestal height in the presence of such energy structures is significantly enhanced when $A_{1}>\sigma_{p_{0}}$.

The spectral pedestal in Fig. 9 is clearly not symmetric about $k_{E}$; there is an satellite region of pedestal at shorter wavelengths. This is because the center of the EEHG 


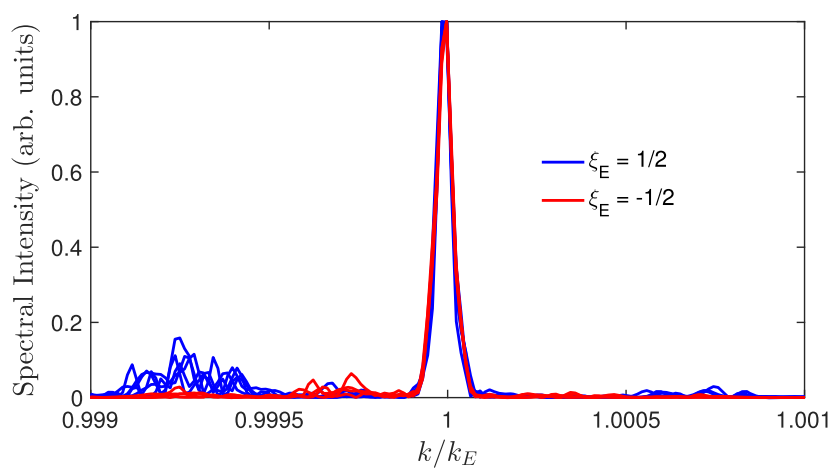

FIG. 10. FEL output spectrum for EEHG with $\xi_{E}<0$ (red) shows reduced spectral pedestal than $\xi_{E}>0$ (blue) on beam with $\sigma_{p_{0}}=\sqrt{3}$ broadband $\mathrm{MBI}$ noise. $A_{1,2}=3, a_{E}=75$, $\rho=1.58 \times 10^{-3}, \sigma_{E} / E=10^{-4}$.

bunching spectrum envelope at $\xi=0$ (where the bunching is also zero), is shifted as usual from $k_{E}$ by $-\xi_{E} k_{1} / B$. The satellite pedestal reflects the shape of the envelopes illustrated in Fig. 7, which plots the optimized envelopes $\left|\bar{b}_{n, m}\right|$ for $\xi_{E}=1 / 2$ and $\xi_{E}=-1 / 2$. Both EEHG tunes generate the same harmonic bunching, but with the $\xi_{E}=$ $1 / 2$ tune, the envelope is redshifted compared to $k_{E}$, whereas for $\xi_{E}=-1 / 2$ it is blue-shifted. Figure 10 shows that this feature may be useful for purifying the FEL output spectrum. The spectrum is the output from multiple 3D simulations with the code PUFFIN [28] on a beam with noisy broadband energy modulations. Results show it is advantageous to choose the EEHG tune with $\xi_{E}<0$ so that most of the sideband pedestal is at higher frequencies, particularly if the FEL bandwidth is less than the excitation bandwidth. This tune takes advantage of the redshifted asymmetry of the FEL gain bandwidth to reduce the portion of the bunching pedestal that is amplified. Note also that certain frequencies can be further suppressed by the $J_{ \pm 1}\left(-\xi\left(k_{ \pm}\right) A_{0}\right)$ term.

\section{Bunching at long wavelengths}

The coherent bunching at the sideband frequencies $k_{E} \pm k_{0}$ is not the only source of FEL pedestal formation. These frequencies are amplified exponentially, but bunching in the electron beam at much longer wavelengths (e.g, at $\lambda_{0}$ ) can also produce a pedestal by parasitically mixing frequencies into the FEL bandwidth that grow even faster during high-gain lasing [13].

In the present model, we can also calculate bunching at the frequency $k_{0}$ generated during transport of the energymodulated beam through the EEHG transformation. It is given by (A5),

$b_{0,0}\left(k_{0}\right)=e^{-\left(\xi^{\prime}\right)^{2} / 2} J_{0}\left(\xi^{\prime} A_{1}\right) J_{0}\left(k_{0} A_{2} B_{2} / k_{1}\right) J_{1}\left(-\xi^{\prime} A_{0}\right)$, where $\xi^{\prime}=k_{0} B / k_{1}$. Inspection shows that the excitation bandwidth is exactly the same as in (26), namely, the bunching at frequencies $k_{0}>2 k_{1} / B$ is strongly damped by the strong dispersion. Assuming $k_{0} \ll k_{1} / B$ and expanding to lowest order, the bunching is peaked near the frequency $k_{0}^{\prime} \approx \frac{k_{1}}{B} \frac{2 \sqrt{2 / 3}}{\sqrt{A_{0}^{2}+2 A_{1}^{2}+4}}$, at which the bunching is,

$$
\left|b_{0,0}\left(k_{0}^{\prime}\right)\right| \approx \frac{A_{0}}{3} \frac{2 \sqrt{2 / 3}}{\sqrt{A_{0}^{2}+2 A_{1}^{2}+4}} .
$$

The analysis in [13] showed that at $\left|b_{0,0}\right| \approx 1 / 3$ the rapidly growing sideband reaches the same power level as the coherently seeded harmonic peak at FEL saturation. This constraint suggests that the modulation amplitude at $k_{0}^{\prime}$ should satisfy,

$$
A_{0}<A_{1}
$$

to keep the FEL sideband power from equaling the harmonic power.

HGHG is essentially insensitive to density modulations produced at long wavelengths in this manner because the required dispersion is too weak to produce significant bunching at $k_{0}$ from the pure energy modulations considered here. The long wavelength energy modulations, however, can also produce sidebands that grow quadratically in the FEL [13]. This phenomena applies to both HGHG and EEHG if the energy modulations survive through the harmonic up-conversion stages, and is a topic of future study.

\section{CONCLUSIONS}

With a simple model we have shown that EEHG and HGHG quantitatively exhibit different sensitivities to a variety of energy modulations imprinted on the electron beam prior to the harmonic up-conversion process. The sensitivities are governed primarily by the respective scaling parameters defined for each scheme, $\xi_{E}$ and $\xi_{H}$, with EEHG being generally less sensitive to both long and intermediate wavelength modulations because typically $\left|\xi_{E}\right|<\xi_{H}$. For this reason the spectral bunching pedestal amplitude in EEHG is also smaller. The pedestal width in each scheme has also been examined and quantified by the intrinsic excitation bandwidth, EB. The strong dispersion required for EEHG leads to strong damping of sideband frequencies and therefore a much narrower EB, which can lead to a cleaner FEL spectrum if it is narrower than the FEL bandwidth. The EEHG bunching envelope may also be partially blue-shifted outside the FEL bandwidth with a $\xi_{E}<0$ tune to further reduce the amplified pedestal. In general, the coherently seeded pedestal in both schemes is smaller the more strongly that $A_{0}<\left|1.4 / \xi_{E, H}\right|$ is satisfied. 


\section{ACKNOWLEDGMENTS}

The authors thank W. M. Fawley, G. Marcus, A. Marinelli, G. Penn, E. Allaria, and P. R. Ribič for helpful comments and discussions. This work was supported in part by the U.S. DOE Office of Basic Energy Sciences under award number 2012-SLAC-10032. The work of D.X. was supported by the Major State Basic Research Development Program of China (Grants No. 2015CB859700) and by the National Natural Science Foundation of China (Grants No. 11327902).

\section{APPENDIX: ANALYTIC DESCRIPTION}

The derivation and notation of [3] for EEHG have been closely followed where applicable. Energy variables are expressed with respect to the mean beam energy and normalized to the slice energy spread, i.e., $p_{i}=\Delta E_{i} / \sigma_{E}$. We consider a finite beam with an initial longitudinal phase space distribution function $f(p, z)=\left(2 \pi \sigma_{z}\right)^{-1} e^{-p^{2} / 2-z^{2} / 2 \sigma_{z}^{2}}$ where $\sigma_{z}$ is the rms length. Prior to the EEHG transformations, the beam is assumed to undergo a general initial energy modulation of the form:

$$
p_{0}=p+A_{0} \sin \left(k_{0} z+\phi\right)-A_{0} \sin (\phi),
$$

where $A_{0}=\Delta E_{0} / \sigma_{E}$ is the normalized energy modulation amplitude, $\lambda_{0}=2 \pi / k_{0}$ is the modulation wavelength, and $\phi$ is the phase. In anticipation of effects due to long wavelength modulations (e.g., wakes or rf chirps), the last term keeps the energy $E$ of electrons in the beam core $(z=0)$ unchanged regardless of the phase.

The beam then enters the EEHG system of two pairs of laser modulators $\left(k_{1,2} \gg k_{0}\right)$ and chicanes. The successive transformations are described by,

$$
\begin{aligned}
p_{1} & =p_{0}+A_{1} \sin \left(k_{1} z\right), \\
z_{1} & =z+B_{1} p_{1} / k_{1}, \\
p_{2} & =p_{1}+A_{2} \sin \left(k_{2} z_{1}\right), \\
z_{2} & =z_{1}+B_{2} p_{2} / k_{1} .
\end{aligned}
$$

Ideal lasers are assumed that completely cover the electron beam both longitudinally and transversally. Phase differences between the two lasers are assumed negligible. The bunching factor at the exit of the last chicane is defined as

$$
b(k)=\int f(p, z) e^{-i k z_{2}(p, z)} \mathrm{d} p \mathrm{~d} z,
$$

In terms of the initial coordinates $(p, z)$ the exponential factor is,

$$
\begin{aligned}
e^{-i k z_{2}(p, z)}= & e^{-i k z} \sum_{n, m=-\infty}^{\infty} e^{i\left(n k_{1}+m k_{2}\right) z-i \xi p_{0}(p, z)} \\
& \times J_{n}\left(-\xi A_{1}\right) J_{m}\left(-k A_{2} B_{2} / k_{1}\right) \\
= & \sum_{q, n, m=-\infty}^{\infty} e^{-i\left(k-q k_{0}-n k_{1}-m k_{2}\right) z} e^{-i \xi p} e^{i q \phi+i \xi A_{0} \sin (\phi)} \\
& \times J_{q}\left(-\xi A_{0}\right) J_{n}\left(-\xi A_{1}\right) J_{m}\left(-k A_{2} B_{2} / k_{1}\right) .
\end{aligned}
$$

The integrals in Eq. (A3) over the initial coordinates are straightforward. The full bunching can be written as a sum of the bunching at the different laser harmonics, $b(k)=\sum_{n, m} b_{n, m}(k)$, where

$$
\begin{aligned}
b_{n, m}(k)= & \bar{b}_{n, m}(k) e^{i \xi A_{0} \sin \phi} \\
& \times \sum_{q=-\infty}^{\infty} e^{i q \phi-\frac{1}{2} \sigma_{z}^{2}\left(k-q k_{0}-n k_{1}-m k_{2}\right)^{2}} J_{q}\left(-\xi A_{0}\right),
\end{aligned}
$$

where the bunching spectrum envelope $\bar{b}_{n, m}(k)$ is defined in Eq. (15). For intermediate wavelength modulations described here, the overall phase of the initial modulation is ignored.

For long wavelength modulations $\lambda_{0} \gg 2 \pi \sigma_{z}$ the first modulation $p_{0}$ is expanded to second order for the linear and quadratic chirps,

$$
p_{0}=p+h_{1} k_{1} z+h_{2} k_{1}^{2} z^{2}
$$

In terms of the initial amplitude and phase of Eq. (A1) we have $h_{1}=A_{0} k_{0} \cos (\phi) / k_{1}$ and $h_{2}=-A_{0} k_{0}^{2} \sin (\phi) / 2 k_{1}^{2}$. Substitution into Eq. (A4b) and calculation of the integral in (A3) yield the bunching spectrum in Eq. (13).

[1] L. H. Yu, Generation of intense uv radiation by subharmonically seeded single-pass free-electron lasers, Phys. Rev. A 44, 5178 (1991).

[2] G. Stupakov, Using the Beam-Echo Effect for Generation of Short-Wavelength Radiation, Phys. Rev. Lett. 102, 074801 (2009).

[3] D. Xiang and G. Stupakov, Echo-enabled harmonic generation free electron laser, Phys. Rev. ST Accel. Beams 12, 030702 (2009).

[4] E. Saldin, E. Schneidmiller, and M. Yurkov, An analytical description of longitudinal phase space distortions in magnetic bunch compressors, Nucl. Instrum. Methods Phys. Res., Sect. A 483, 516 (2002), proceedings of the 23rd International Free Electron Laser Conference and 8th $\{$ FEL $\}$ Users Workshop. 
[5] S. Heifets, G. Stupakov, and S. Krinsky, Coherent synchrotron radiation instability in a bunch compressor, Phys. Rev. ST Accel. Beams 5, 064401 (2002).

[6] Z. Huang and K.-J. Kim, Formulas for coherent synchrotron radiation microbunching in a bunch compressor chicane, Phys. Rev. ST Accel. Beams 5, 074401 (2002).

[7] K. L. F. Bane, F.-J. Decker, Y. Ding, D. Dowell, P. Emma, J. Frisch, Z. Huang, R. Iverson, C. Limborg-Deprey, H. Loos et al., Measurements and modeling of coherent synchrotron radiation and its impact on the Linac Coherent Light Source electron beam, Phys. Rev. ST Accel. Beams 12, 030704 (2009).

[8] E. Saldin, E. Schneidmiller, and M. Yurkov, Longitudinal space charge-driven microbunching instability in the TESLA Test Facility linac, Nucl. Instrum. Methods Phys. Res., Sect. A 528, 355 (2004), proceedings of the 25th International Free Electron Laser Conference, and the 10th FEL Users Workshop.

[9] Z. Huang, M. Borland, P. Emma, J. Wu, C. Limborg, G. Stupakov, and J. Welch, Suppression of microbunching instability in the linac coherent light source, Phys. Rev. ST Accel. Beams 7, 074401 (2004).

[10] M. Venturini, Models of longitudinal space-charge impedance for microbunching instability, Phys. Rev. ST Accel. Beams 11, 034401 (2008).

[11] D. Ratner, R. Abela, J. Amann, C. Behrens, D. Bohler, G. Bouchard, C. Bostedt, M. Boyes, K. Chow, D. Cocco et al., Experimental Demonstration of a Soft X-Ray Self-Seeded Free-Electron Laser, Phys. Rev. Lett. 114, 054801 (2015).

[12] J. Amann, W. Berg, V. Blank, F.-J. Decker, Y. Ding, P. Emma, Y. Feng, J. Frisch, D. Fritz, J. Hastings et al., Demonstration of self-seeding in a hard-X-ray freeelectron laser, Nat. Photonics 6, 693 (2012).

[13] Z. Zhang, R. Lindberg, W. M. Fawley, Z. Huang, J. Krzywinski, A. Lutman, G. Marcus, and A. Marinelli, Microbunching-instability-induced sidebands in a seeded free-electron laser, Phys. Rev. Accel. Beams 19, 050701 (2016).

[14] Z. Huang, D. Ratner, G. Stupakov, and D. Xiang, in Proceedings of the 31st International Free Electron Laser Conference (FEL 09), Liverpool, UK (STFC Daresbury Laboratory, Warrington, 2009).

[15] E. Hemsing, M. Dunning, C. Hast, T. O. Raubenheimer, S. Weathersby, and D. Xiang, Highly coherent vacuum ultraviolet radiation at the 15 th harmonic with echo-enabled harmonic generation technique, Phys. Rev. ST Accel. Beams 17, 070702 (2014).

[16] G. Stupakov, Report No. SLAC-PUB-14639, 2011.

[17] G. Penn, Stable, coherent free-electron laser pulses using echo-enabled harmonic generation, Phys. Rev. ST Accel. Beams 17, 110707 (2014).

[18] G. Penn, P. Emma, E. Hemsing, Z. Huang, G. Marcus, J. Qiang, T. Raubenheimer, and L. Wang, in Proceedings of FEL 2015, Daejeon, Korea (JACoW, Geneva, Switzerland, 2015).

[19] P. Rebernik Ribič, E. Roussel, G. Penn, G. De Ninno, L. Giannessi, G. Penco, and E. Allaria, Echo-enabled harmonic generation studies for the FERMI free-electron laser, Photonics Spectra 4, 19 (2017).

[20] Z. T. Zhao, D. Wang, J. H. Chen, Z. H. Chen, H. X. Deng, J. G. Ding, C. Feng, Q. Gu, M. M. Huang, T. H. Lan et al., First lasing of an echo-enabled harmonic generation freeelectron laser, Nat. Photonics 6, 360 (2012).

[21] E. Roussel, E. Ferrari, E. Allaria, G. Penco, S. Di Mitri, M. Veronese, M. Danailov, D. Gauthier, and L. Giannessi, Multicolor High-Gain Free-Electron Laser Driven by Seeded Microbunching Instability, Phys. Rev. Lett. 115, 214801 (2015).

[22] G. Penn, LBNL Report No. NGLS Technical Note 35, 2012.

[23] C. Feng, D. Wang, and Z. T. Zhao, in Proceedings of the 3rd International Particle Accelerator Conference, New Orleans, LA, 2012 (IEEE, Piscataway, NJ, 2012), p. 1724.

[24] T. Shaftan and L. H. Yu, High-gain harmonic generation free-electron laser with variable wavelength, Phys. Rev. E 71, 046501 (2005).

[25] MATLAB, version 9.0.0.341360 (R2016a) (The MathWorks Inc., Natick, Massachusetts, 2016).

[26] R. Bonifacio, C. Pellegrini, and L. M. Narducci, Collective instabilities and high-gain regime in a free electron laser, Opt. Commun. 50, 373 (1984).

[27] D. Ratner, C. Behrens, Y. Ding, Z. Huang, A. Marinelli, T. Maxwell, and F. Zhou, Time-resolved imaging of the microbunching instability and energy spread at the Linac Coherent Light Source, Phys. Rev. ST Accel. Beams 18, 030704 (2015).

[28] L. T. Campbell and B. W. J. McNeil, Puffin: A three dimensional, unaveraged free electron laser simulation code, Phys. Plasmas 19, 093119 (2012). 